\title{
Evaluation of the specific combining ability (SCA) for yield and brix of seven Cucumis melo. L inbred lines of the sixth generation
}

\author{
Tuan Q. Huynh ${ }^{1 *}$, Hiet D. Hoang ${ }^{1}, \operatorname{Trinh}$ T. T. To ${ }^{1}, \operatorname{Lam}_{\text {V. }} \operatorname{Tran}^{1}$, \\ Man T. T. Le ${ }^{1}$, \& Dan T. Vo ${ }^{2}$ \\ ${ }^{1}$ Research and Development Center for Hi-tech Agriculture, Ho Chi Minh City, Vietnam \\ ${ }^{2}$ Faculty of Agronomy, Nong Lam University, Ho Chi Minh City, Vietnam
}

ARTICLE INFO
Research Paper
Received: December 08, 2020
Revised: April 09, 2021
Accepted: April 23, 2021
Keywords
Brix
Cucumis melo L.
Specific combining ability (SCA)
Yield

${ }^{*}$ Corresponding author

Huynh Quang Tuan

Email: hqt.org@gmail.com

\section{ABSTRACT}

The objective of the experiment was to screen Cucumis melo L. inbred lines for high potential general combining ability and potential hybrids combinations for F1 hybridization. The experiment was conducted as a randomized complete block design (RCBD) with 29 treatments [21 hybrid combinations, control (TL3 hybrid) and seven Cucumis melo L. inbred lines $\left.\mathrm{I}_{6}\right]$, each with three replicates. Estimating the specific combining ability (SCA) of Cucumis melo L. inbred lines for breeding of F1 hybrid cultivars was carried out at the Research and Development Center for Hi-tech Agriculture in Ho Chi Minh City. Based on the specific combining ability of yield trait and total soluble solids (Brix) content of 21 hybrid lines from seven inbred lines following diallel method (Griffing 4 model), four potential hybrid lines (H41.6 x H58.6, H53.6 x H77.6, H32.6 x H41.6 và H53.6 x H58.6) yielded $10 \%$ higher than the control variety and gave fruits of oval shape, even fruit-net and low diseases infection.

Cited as: Huynh, T. Q., Hoang, H. D., To, T. T. T., Tran, L. V., Le, M. T. T., \& Vo, D. T. (2021). Evaluation of the specific combining ability (SCA) for yield and brix of seven Cucumis melo. L inbred lines of the sixth generation. The Journal of Agriculture and Development 20(2), 1-9. 


\title{
Đánh giá khả năng phối hợp riêng về năng suất và độ brix của bảy dòng dưa lưới (Cucumis melo L.) thế hệ thứ sáu $\left(\mathrm{I}_{6}\right)$
}

\author{
Huỳnh Quang Tuấn ${ }^{1 *}$, Hoàng Đắc Hiệt ${ }^{1}$, Tô Thị Thùy Trinh ${ }^{1}$, Trần Văn Lâm ${ }^{1}$, \\ Lê Thị Thu Mận ${ }^{1} \&$ Võ Thái Dân ${ }^{2}$ \\ ${ }^{1}$ Trung Tâm Nghiên Cứu và Phát Triển Nông Nghiệp Công Nghệ Cao, TP. Hồ Chí Minh \\ ${ }^{2}$ Khoa Nông Học, Trường Đại Học Nông Lâm TP.HCM, TP. Hồ Chí Minh
}

THÔNG TIN BÀI BÁO
Bài báo khoa học
Ngày nhận: 08/12/2020
Ngày chỉnh sửa: 09/04/2021
Ngày chấp nhận: 23/04/2021
Từ khóa
Dưa lưới
Độ brix
Khả năng phối hợp riêng (SCA)
Năng suất

*Tác giả liên hệ

Huỳnh Quang Tuấn

Email: hqt.org@gmail.com

\section{TÓM TẮT}

Mục tiêu là chọn các dòng dưa lưới có khả năng kết hợp riêng cao và các tổ hợp lai dưa lưới ưu tú phục vụ lai tạo giống F1. Thí nhiệm đơn yếu tố, được bố trí theo kiểu khối hoàn toàn ngẫu nhiên gồm 29 nghiệm thức (21 tổ hợp lai, 1 giống đối chứng (giống lai TL3) và 7 dòng dưa lưới $\mathrm{I}_{6}$ ) và 3 lần lặp lại. Đánh giá khả năng phối hợp riêng dựa trên tính trạng năng suất và độ brix của 7 dòng dưa lưới tự phối $\mathrm{I}_{6}$ (H5.6, H32.6, H34.6, H41.6, H53.6, H58.6, H77.6) bằng phương pháp lai luân giao (mô hình Griffing 4) được thực hiện tại Trung tâm Nghiên cứu và Phát triển Nông nghiệp Công nghệ cao, chọn được 04 tổ hợp lai (THL) triển vọng có khả năng phối hợp riêng và ưu thế lai cao vượt giống đối chứng, phục vụ công tác sản xuất gồm THL H41.6 x H58.6, H53.6 x H77.6, H32.6 x H41.6 và H53.6 x H58.6. Ngoài khả năng có ưu thế lai và cho năng suất cao hơn giống đối chứng $10 \%$, bốn THL này còn thể hiện các đặc điểm hình thái như quả tròn, lưới đều và ít nhiễm sâu bệnh.

\section{1. Đặt Vấn Đề}

Sản xuất dưa lưới thương phẩm phổ biến ở Việt Nam khoảng từ năm 2008 - 2010. Mặc dù quy mô sản xuất vẫn còn rất nhỏ lẻ, song trồng dưa lưới theo mô hình nông nghiệp công nghệ cao rất hiệu quả và có thể nhân rộng (Ngoc, 2020). Hiện nay, phần lớn các giống dưa lưới đưa vào sản xuất đều có nguồn gốc từ nước ngoài như Hà Lan, Israel, Nhật Bản, Đài Loan, Thái Lan. Giá thành hạt giống cao và chi phí sản xuất lớn làm cho sản phẩm sản xuất được có giá thành khá đắt, chưa phổ biến rộng cho người tiêu dùng. Để có thể mở rộng đối tượng khách hàng tiêu thụ dưa lưới, việc xem xét hạ giá thành sản phẩm là hết sức cần thiết. Bên cạnh nghiên cứu các biện pháp kỹ thuật sản xuất thích hợp để đạt năng suất cao, chất lượng tốt, vấn đề chọn tạo giống tiến tới chủ động được nguồn hạt giống và không phụ thuộc vào nguồn giống nhập nội; phù hợp với điều kiện khí hậu Việt Nam được xem là bước đột phá.

Nhận thấy tiềm năng của thị trường hạt giống trong nước từ năm 2016 - 2019, Trung tâm Nghiên cứu và Phát triển Nông nghiệp Công nghệ cao đã thu thập và chọn tạo kết quả đến năm 2019 đã chọn được 7 dòng dưa lưới thế hệ $\mathrm{I}_{6}$ (Huynh \& ctv., 2019). Đánh giá khả năng phối hợp riêng để chọn ra các tổ hợp lai (THL) ưu tú là bước quan trọng tiếp theo trong quá trình sản xuất hạt giống lai F1. Mục tiêu của nghiên cứu là chọn được THL có năng suất và độ brix tương đương hoặc vượt trội so với giống dưa lưới F1 đang được sản xuất trên thị trường.

\section{Vật Liệu và Phương Pháp Nghiên Cứu}

\subsection{Vật liệu}

Tổ hợp lai: + 21 THL (H5.6 x H77.6, H32.6 x H34.6, H32.6 x H53.6, H34.6 x H53.6, H34.6 
x H58.6, H41.6 x H53.6, H41.6 x H58.6, H41.6 x $\mathrm{H} 77.6, \mathrm{H} 53.6$ x H58.6 và H53.6 x H77.6, H5.6 x H32.6, H5.6 x H34.6, H5.6 x H41.6, H5.6 x H53.6, H5.6 x H58.6, H32.6 x H41.6, H32.6 x H58.6, H32.6 x H77.6, H34.6 x H41.6, H34.6 x H77.6 và $\mathrm{H} 58.6$ x H77.6) được tạo bằng phương pháp lai luân giao (mô hình Griffing 4) bởi 7 dòng dưa lưới thế hệ $\mathrm{I}_{6}$, được Trung tâm Nghiên cứu và Phát triển Nông nghiệp Công nghệ cao chọn lọc trong giai đoạn 2016 - 2019 (Bảng 1).

Giống đối chứng TL3: giống lai F1, của công ty Chánh Phong.

Giá thể:

Mụn dừa được xử lý sạch chất chát bằng cách ngâm xả nước trong 7 - 10 ngày.

Phân trùn quế: Chất hữu cơ (\%): C: 7,64; mùn: 13,17; chất tổng số (\%): $\mathrm{N}: 1,93: \mathrm{P}: 0,71 ; \mathrm{K}: 0,70$ (AHRD, 2015).

Tro trấu.

Tỷ lệ phối trộn giá thể: $70 \%$ mụn dừa: $20 \%$ phân trùn quế: $10 \%$ tro trấu.

Các loại hóa chất pha dung dịch tưới: $\mathrm{KH}_{2} \mathrm{PO}_{4}$ $(0,25 \mathrm{~g} / \mathrm{L}), \mathrm{Ca}\left(\mathrm{NO}_{3}\right)_{2} \cdot 4 \mathrm{H}_{2} \mathrm{O}(1,115 \mathrm{~g} / \mathrm{L}), \mathrm{K}_{2} \mathrm{SO}_{4}$ $(0,2 \mathrm{~g} / \mathrm{L}), \mathrm{MgSO}_{4} \cdot 7 \mathrm{H}_{2} \mathrm{O}(0,55 \mathrm{~g} / \mathrm{L}), \mathrm{H}_{3} \mathrm{BO}_{3}(0,5$ $\mathrm{mg} / \mathrm{L}), \mathrm{ZnSO}_{4}(\mathrm{Zn:} 0,3 \mathrm{mg} / \mathrm{L}), \mathrm{CuSO}_{4} .5 \mathrm{H}_{2} \mathrm{O}(0,5$ $\mathrm{mg} / \mathrm{L}), \mathrm{Na}_{2} \mathrm{MoH}_{2} \mathrm{O}(0,05 \mathrm{mg} / \mathrm{L})$ và Chelate sắt (3 mg/L).

Thí nghiệm được thực hiện trong điều kiện nhà màng kiểu máy thông gió cố định, dưa lưới được trồng trên giá thể, nước và phân bón được tưới qua hệ thống tưới nhỏ giọt.

\subsection{Phương pháp nghiên cứu}

Thí nghiệm đơn yếu tố được bố trí theo kiểu khối hoàn toàn ngẫu nhiên (RCBD), 29 nghiệm thức (21 THL, 1 giống đối chứng (TL3) và 7 dòng bố mẹ I6) và ba lần lặp lại. Mỗi ô thí nghiệm trồng thành 2 hàng với 50 cây, có diện tích ô là $15 \mathrm{~m}^{2}$, tổng số cây cho một nghiệm thức là 150 cây/nghiệm thức; khoảng cách trồng hàng x hàng $=0,6 \mathrm{~m}$; cây x cây $=0,5 \mathrm{~m}$.

Hạt dưa lưới được gieo vào ngày 4 tháng 09 , trồng vào ngày 14 tháng 09 và thu hoạch từ ngày 11 đến ngày 15 tháng 11 năm 2019. Quy cách cây con: Chiều cao cây: 12 - $15 \mathrm{~cm}$; số lá thật: 2 - 3 lá.

Các chỉ tiêu theo dõi: Tỷ lệ đậu quả (\%); Hình dạng quả; Màu sắc vỏ quả; Màu sắc thịt quả; Đặc điểm vân lưới; Khối lượng quả (kg/quả); Năng suất thực thu (tấn/1000 $\mathrm{m}^{2}$ ); Độ brix thịt quả (\%); Tỷ lệ bệnh hại (\%): bệnh thối thân (Didymella bryoniae), giả sương mai (Pseudoperonospora cubensis), phấn trắng (Erysiphe cichoracearum), héo xanh (Pseudomonas solanacearum), xì mủ (Mycosphaerella melonis) (MARD, 2003).

Đánh giá khả năng phối hợp riêng trên 2 chỉ tiêu: Năng suất thực thu (tấn/1000 m²); Độ brix thịt quả (\%).

Nội dung nghiên cứu được tiến hành trong điều kiện nhà màng, cây dưa lưới được chăm sóc theo quy trình trồng dưa lưới trong nhà màng tại Trung tâm Nghiên cứu và Phát triển Nông nghiệp Công nghệ cao (MARD, 2014).

Phương pháp đánh giá khả năng phối hợp riêng theo phương pháp mô hình Griffing 4 (Griffing, 1956).

Mô hình toán học chung:

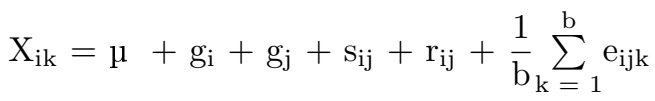

Trong đó:

$\mathrm{X}_{\mathrm{ik}}$ : Độ lớn tính trạng con lai ( $\mathrm{ix}_{\mathrm{j}}$ ) ở lần nhắc thứ $\mathrm{k}$

$\mu$ : Tính trạng trung bình trong thí nghiệm

$g_{i}$ và $g_{j}$ : Khả năng kết hợp chung của dòng i và dòng $\mathrm{j}$

$\mathrm{s}_{\mathrm{ij}}$ : Tác động của khả năng kết hợp riêng giữa hai dòng $I$ và dòng $\mathrm{j}$

$\mathrm{r}_{\mathrm{ij}}$ : Tác động tương hỗ giữa 2 dòng đó

$\mathrm{e}_{\mathrm{ijk}}$ : Sai số ngẫu nhiên

Xác định mức độ ưu thế lai: Ưu thế lai trung bình (HM\%), Ủu thế lai thực (HB\%), Ưu thế lai chuẩn (HS\%) (Phan, 2006).

Ưu thế lai trung bình là ưu thế lai mà các tính trạng hình thái và năng suất cao hơn trung bình, giữa hai bố mẹ hoặc vượt trội cả hai bố mẹ:

$$
\mathrm{HM}, \%=\frac{\mathrm{F} 1-\mathrm{MP}}{\mathrm{MP}} \times 100
$$

Ưu thế lai thực (HB) là ưu thế lai khi so với bố mẹ tốt nhất:

$$
\mathrm{HB}, \%=\frac{\mathrm{F} 1-\mathrm{BP}}{\mathrm{BP}} \times 100
$$

Ưu thế lai chuẩn (HS) là ưu thế lai biểu thị sự hơn kém của con lai $\mathrm{F} 1$ so với giống đối chứng trong sản xuất đại trà:

$$
\mathrm{HS}, \%=\frac{\mathrm{F} 1-\mathrm{S}}{\mathrm{S}} \times 100
$$

Trong đó: 
Bảng 1. Đặc điểm hình thái quả, năng suất và độ brix của 7 dòng dưa lưới thế hệ I6 và giống đối chứng TL3

\begin{tabular}{cccccccc}
\hline $\begin{array}{c}\text { Tên } \\
\text { dòng }\end{array}$ & $\begin{array}{c}\text { Hình } \\
\text { dạng quả }\end{array}$ & $\begin{array}{c}\text { Màu vỏ } \\
\text { quả }\end{array}$ & $\begin{array}{c}\text { Đặc điểm } \\
\text { vân lưới }\end{array}$ & $\begin{array}{c}\text { Màu thịt } \\
\text { quả }\end{array}$ & $\begin{array}{c}\text { Khối } \\
\text { lượng quả } \\
(\mathrm{kg} / \text { quả })\end{array}$ & $\begin{array}{c}\text { Năng suất } \\
(\text { tấn/1000 } \\
\left.\mathrm{m}^{2}\right)\end{array}$ & $\begin{array}{c}\text { Độ brix } \\
(\%)\end{array}$ \\
\hline H5.6 & Tròn & Xanh & Dày, đều & Xanh & 1,03 & 1,70 & 13,33 \\
H32.6 & Trứng & Xanh & Dày, đều & Cam & 1,40 & 1,93 & 15,33 \\
H34.6 & Trứng & Xanh & Dày, đều & Cam & 1,10 & 1,83 & 12,33 \\
H41.6 & Tròn & Xanh & Dày & Xanh & 0,90 & 1,30 & 14,66 \\
H53.6 & Trứng & Xanh & Thưa, đều & Cam & 1,47 & 2,37 & 14,33 \\
H58.6 & Trứng & Xanh & Thưa, đều & Cam & 1,07 & 1,77 & 14,33 \\
H77.6 & Tròn & Vàng & Dày & Xanh & 1,07 & 1,90 & 12,33 \\
TL3 & Tròn & Xanh & Dày, đều & Xanh & 1,57 & 3,06 & 13,00 \\
\hline
\end{tabular}

\section{F1: giá trị con lai F1}

MP: giá trị trung bình của bố mẹ

BP: là giá trị bố mẹ tốt nhất

S: giá trị giống đối chứng

Xử lý số liệu: Số liệu thí nghiệm được phân tích ANOVA, phân hạng trên phần mềm SAS 9.1; khả năng phối hợp riêng được tính toán trên phần mềm Microsoft Excel.

\section{Kết Quả và Thảo Luận}

3.1. Đặc điểm hình thái, năng suất và chất lượng 21 THL từ bay dòng dưa lưới thế hệ $\mathbf{I}_{6}$

Tỷ lệ đậu quả là một chỉ tiêu quan trọng trong sản xuất dưa lưới, theo kết quả của nghiên cứu cho thấy 21 THL từ 7 dòng dưa lưới đều cho tỷ lệ đậu quả cao, trung bình đạt 99,8\%, riêng tổ hợp lai H41.6 x H58.6 và H53.6 x H77.6 có tỷ lệ đậu quả thấp hơn là $97 \%$ và $98 \%$. Tỷ lệ đậu quả của giống đối chứng (TL3) là 100\%. Tỷ lệ đậu quả ở mức cao, điều này phù hợp với nhu cầu chọn tạo giống dưa lưới F1.

Các THL có hình dạng quả và màu sắc vỏ quả đặc trưng, qua khảo sát đặc điểm bên ngoài của 21 THL từ bảy dòng dưa lưới thế hệ I6, kết quả cho thấy có hai dạng kiểu hình về hình dạng quả là hình trứng và hình tròn. Các THL có dạng quả hình trứng gồm H5.6 x H77.6, H32.6 x H34.6, H32.6 x H53.6, H34.6 x H53.6, H34.6 x H58.6, $\mathrm{H} 41.6 \times \mathrm{H} 53.6$, H41.6 x H58.6, H41.6 x H77.6, H53.6 x H58.6 và H53.6 x H77.6). Các THL có dạng quả hình tròn là H5.6 x H32.6, H5.6 x H34.6, H5. 6 x H41.6, H5.6 x H53.6, H5.6 x H58.6, H32.6 x H41.6, H32.6 x H58.6, H32.6 x H77.6, H34.6 x H41.6, H34.6 x H77.6 và H58.6 x H77.6. Trong 21 THL dưa lưới có sáu THL có vỏ quả màu vàng
(H5.6 x H77.6, H32.6 x H77.6, H34.6 x H77.6, H41.6 x H77.6, H53.6 x H77.6 và H58.6 x H77.6), còn các THL còn lại có vỏ quả màu xanh. Về chỉ tiêu thịt quả của 21 THL dưa lưới gồm 09 THL có thịt quả màu xanh (H5.6 x H41.6, H5.6 x H77.6, H32. 6 x H41.6, H32.6 x H77.6, H34.6 x H41.6, H34.6 x H77.6, H41.6 x H77.6, H53.6 x H77.6 và H58.6 x H77.6) và 12 THL còn lại có thị quả màu cam. Về chỉ tiêu vân lưới phân bố theo kiểu dày có 10 THL gồm H5.6 x H32.6, H5.6 x H34.6, H5.6 x H41.6, H5.6 x H53.6, H32.6 x H34.6, H32.6 x H53.6, H34.6 x H41.6, H34.6 x H53.6, H41.6 x H53.6, H41.6 x H58.6 ) và 11 THL còn lại có vân lưới thưa (Hình 1).

Khối lượng quả là yếu tố quan trọng quyết định năng suất và ảnh hưởng đến giá thành của sản phẩm. Khối lượng quả của các THL dao động trong khoảng 1,23 dến 1,90 kg (Bảng 2). Tổ hợp lai H41.6 x H58.6 (1,90 kg/quả), H53.6 x H77.6 (1,83 kg/quả) là các THL cho khối lượng quả trung bình cao hơn so với đối chứng TL3 $(1,57$ $\mathrm{kg}$ /quả).

Năng suất thực thu của các THL dao động từ 2,39 đến 3,82 tấn $/ 1000 \mathrm{~m}^{2}$, trong đó THL H41.6 x H58.6 (3,82 tấn/1000 m²), H53.6 x H77.6 (3,58 tấn/1000 $\left.\mathrm{m}^{2}\right)$ có năng suất cao hơn đối chứng TL3 (3,06 tấn/1000 $\left.\mathrm{m}^{2}\right)$.

Độ ngọt của dưa lưới là một chỉ tiêu rất quan trọng đối với thị hiếu người tiêu dùng. Độ brix của các THL khác biệt có ý nghĩa về mặt thống kê và dao động trong khoảng từ 9,50 đến $14,87 \%$. So với giống đối chứng TL3 (13,00\%), 4 THL (H32.6 x H53.6, H34.6 x H58.6, H34.6 x H77.6 và H41.6 x H58.6) có độ brix tương đương và 6 THL (H5.6 x H34.6, H32.6 x H41.6, H34.6 x H53.6, H41.6 x H77.6, H53.6 x H58.6 và H53.6 x H77.6) có độ brix cao hơn hẳn so với đối chứng.

Theo đánh giá phân loại dưa lưới (Trung tâm 

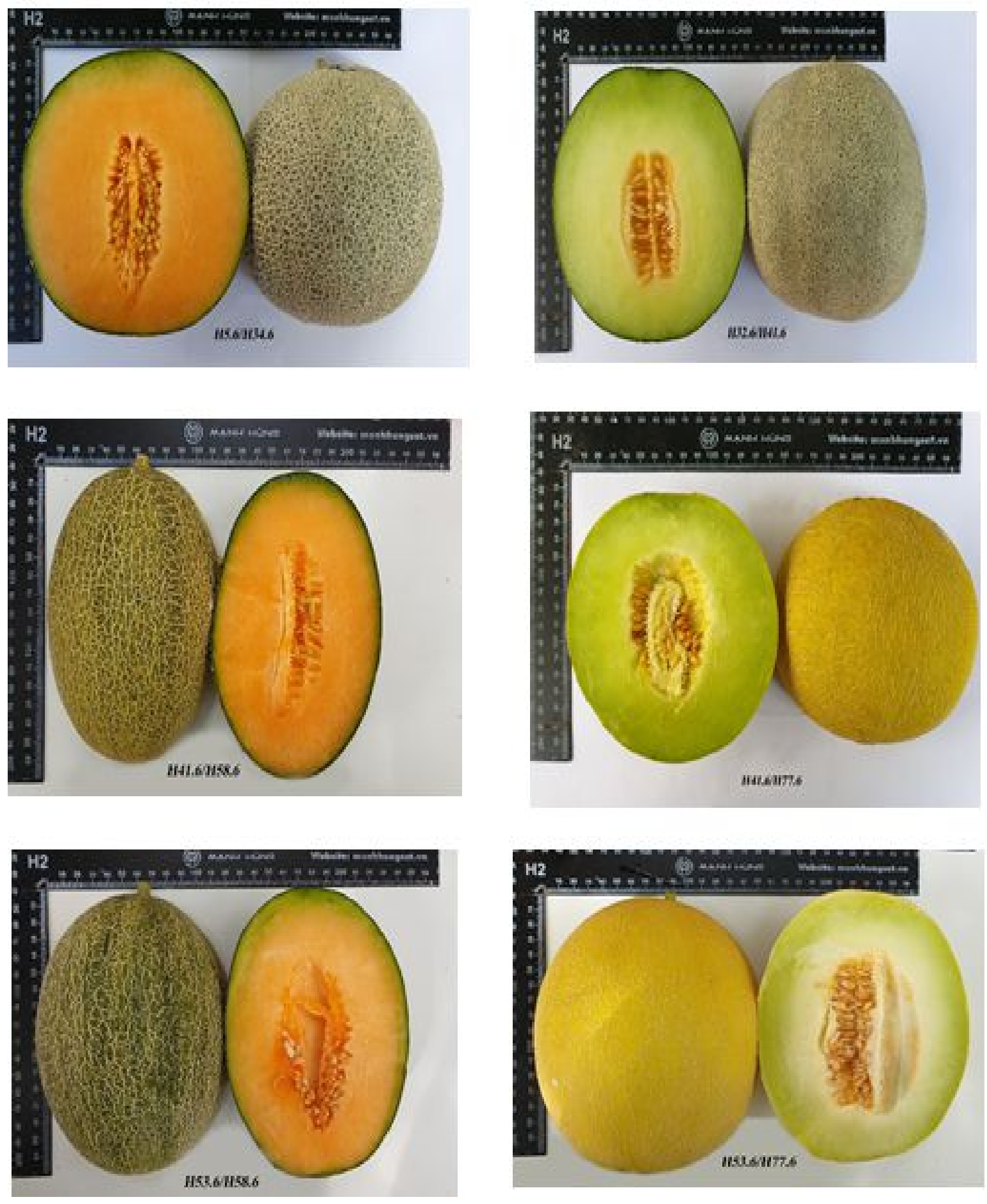

Hình 1. Đặc điểm quả của một số tổ hợp lai từ bảy dòng dưa lưới thế hệ $\mathrm{I}_{6}$.

Nghiên cứu và Phát triển Nông nghiệp Công nghệ cao), dưa lưới loại 1: lưới đều, đẹp, quả 1,2 - 2,5 $\mathrm{kg}$, độ brix 12 - 18; loại 2: lưới đều, đẹp, quả dưới $1,2 \mathrm{~kg}$ hoặc trên $2,5 \mathrm{~kg}$, độ brix 12 - 18 thì các
THL đều đáp ứng về khối lượng là dưa lưới loại 1, về độ brix phần lớn các THL đáp ứng (độ brix trên $12 \%$ ), trong đó kể đến H5.6 x H34.6, H32.6 x H41.6, H34.6 x H53.6, H41.6 x H77.6, H53.6 x 
Bảng 2. Đặc điểm năng suất và độ brix của 21 tổ hợp lai từ bảy dòng dưa lưới thế hệ $\mathrm{I}_{6}$

\begin{tabular}{|c|c|c|c|c|}
\hline $\begin{array}{c}\text { Cặp lai } \\
\left(\sigma^{\top} \times \text { o }\right) / \text { dòng }\end{array}$ & Tỷ lệ đậu quả (\%) & $\begin{array}{l}\text { Khối lượng quả } \\
\text { (kg/quả) }\end{array}$ & $\begin{array}{l}\text { Năng suất thực thu } \\
\left.\text { (tấn } / 1000 \mathrm{~m}^{2}\right)\end{array}$ & Độ brix (\%) \\
\hline $\mathrm{H} 5.6 \times \mathrm{H} 32.6$ & 100 & $1,60^{\mathrm{cb}}$ & $3,19^{\mathrm{cb}}$ & $10,73^{\mathrm{gh}}$ \\
\hline H5.6 x H34.6 & 100 & $1,53^{\mathrm{b}-\mathrm{e}}$ & $3,02^{\mathrm{b}-\mathrm{e}}$ & $14,50^{\mathrm{ab}}$ \\
\hline H5.6 x H41.6 & 100 & $1,47^{\mathrm{d}-\mathrm{g}}$ & $2,88^{\mathrm{def}}$ & $11,83^{\mathrm{e}}$ \\
\hline H5.6 x H53.6 & 100 & $1,40^{\text {fgh }}$ & $2,74^{\mathrm{efg}}$ & $12,00^{\mathrm{e}}$ \\
\hline H5.6 x H58.6 & 100 & $1,43^{\mathrm{efg}}$ & $2,81^{\mathrm{d}-\mathrm{g}}$ & $9,50^{\mathrm{j}}$ \\
\hline H5.6 x H77.6 & 100 & $1,37^{\mathrm{gh}}$ & $2,67^{\text {fgh }}$ & $10,33^{\text {hi }}$ \\
\hline H32.6 x H34.6 & 100 & $1,40^{\text {fgh }}$ & $2,74 \mathrm{e}^{\mathrm{fg}}$ & $11,00^{\text {fg }}$ \\
\hline $\mathrm{H} 32.6 \times \mathrm{H} 41.6$ & 100 & $1,57^{\mathrm{bcd}}$ & $3,09^{\mathrm{bcd}}$ & $14,87^{\mathrm{a}}$ \\
\hline H32.6 x H53.6 & 100 & $1,50^{\mathrm{c}-\mathrm{f}}$ & $2,95^{\mathrm{c}-\mathrm{f}}$ & $13,10^{\mathrm{d}}$ \\
\hline H32.6 x H58.6 & 100 & $1,57^{\mathrm{bcd}}$ & $3,09^{\mathrm{cbd}}$ & $11,00^{\text {fg }}$ \\
\hline H32.6 x H77.6 & 100 & $1,23^{\mathrm{i}}$ & $2,39^{\mathrm{h}}$ & $10,00^{\mathrm{ij}}$ \\
\hline H34.6 x H41.6 & 100 & $1,30^{\mathrm{hi}}$ & $2,53^{\mathrm{gh}}$ & $9,50^{\mathrm{j}}$ \\
\hline H 34.6 x H53.6 & 100 & $1,40^{\mathrm{fgh}}$ & $2,74 \mathrm{e}^{\mathrm{fg}}$ & $14,00^{\mathrm{bc}}$ \\
\hline H34.6 x H58.6 & 100 & $1,47^{\mathrm{d}-\mathrm{g}}$ & $2,91^{\mathrm{c}-\mathrm{f}}$ & $13,17^{\mathrm{d}}$ \\
\hline H34.6 x H77.6 & 100 & $1,50^{\mathrm{c}-\mathrm{f}}$ & $2,98^{\mathrm{b}-\mathrm{e}}$ & $13,50^{\mathrm{cd}}$ \\
\hline H41.6 x H53.6 & 100 & $1,53^{\mathrm{b}-\mathrm{e}}$ & $3,05^{\mathrm{bcd}}$ & $10,50^{\text {ghi }}$ \\
\hline $\mathrm{H} 41.6$ x H58.6 & 97 & $1,90^{\mathrm{a}}$ & $3,82^{\mathrm{a}}$ & $13,50^{\mathrm{cd}}$ \\
\hline $\mathrm{H} 41.6$ x H77.6 & 100 & $1,63^{\mathrm{b}}$ & $3,26^{\mathrm{b}}$ & $14,00^{\mathrm{bc}}$ \\
\hline H53.6 x H58.6 & 100 & $1,63^{\mathrm{b}}$ & $3,26^{\mathrm{b}}$ & $14,50^{\mathrm{ab}}$ \\
\hline H53.6 x H77.6 & 98 & $1,83^{\mathrm{a}}$ & $3,58^{\mathrm{a}}$ & $13,83^{\mathrm{bc}}$ \\
\hline H58.6 × H77.6 & 100 & $1,50^{\mathrm{c}-\mathrm{f}}$ & $2,88^{\mathrm{cf}}$ & $11,50^{\mathrm{ef}}$ \\
\hline TL3 (ĐC) & 100 & $1,57^{\mathrm{cbd}}$ & $3,06^{\mathrm{bcd}}$ & $13,00^{\mathrm{d}}$ \\
\hline H5.6 & 96 & 1,03 & 1,70 & 13,33 \\
\hline Н32.6 & 90 & 1,40 & 1,93 & 15,33 \\
\hline Н34.6 & 100 & 1,10 & 1,83 & 12,33 \\
\hline H41.6 & 100 & 0,90 & 1,30 & 14,66 \\
\hline H53.6 & 97 & 1,47 & 2,37 & 14,33 \\
\hline H58.6 & 95 & 1,07 & 1,77 & 14,33 \\
\hline H77.6 & 100 & 1,07 & 1,90 & 12,33 \\
\hline $\mathrm{CV}(\%)$ & & 3,46 & 4,39 & 2,67 \\
\hline $\mathrm{LSD}_{0,01}$ & & 0,12 & 0,29 & 0,54 \\
\hline
\end{tabular}

a-g Trong cùng một cột, các giá trị trung bình có cùng ký tự đi kèm không khác biệt ở mức $P<0,01$.

Chỉ phân hạng trên 21 THL và đối chứng.

21 THL: H5.6 x H77.6, H32.6 x H34.6, H32.6 x H53.6, H34.6 x H53.6, H34.6 x H58.6, H41.6 x H53.6, H41.6 x H58.6, H41.6 x H77.6, H53.6 x H58.6 và H53.6 x H77.6, H5.6 x H32.6, H5.6 x H34.6, H5.6 x H41.6, H5.6 x H53.6, H5.6 x H58.6, H32.6 x $\mathrm{H} 41.6, \mathrm{H} 32.6$ x H58.6, H32.6 x H77.6, H34.6 x H41.6, H34.6 x H77.6 và H58.6 x H77.6.

Bảy dòng dưa lưới thế hệ I6: H5.6, H32.6, H34.6, H41.6, H53.6, H58.6, H77.6.

\section{H58.6 và H53.6 x H77.6.}

Để chọn lọc các THL, ngoài chỉ tiêu về năng suất và độ brix cao thì tỷ lệ nhiễm sâu bệnh của các THL phải ở mức thấp. Tỷ lệ nhiễm sâu bệnh càng thấp thì sẽ ít ảnh hưởng đến năng suất, đây là một chỉ tiêu mà người tiêu dùng rất quan tâm khi lựa chọn giống dưa lưới để sản xuất.

Đối với các THL ở thế hệ $\mathrm{I}_{6}$, các đối tượng nhiễm bệnh chủ yếu: bệnh thối thân (Didymella bryoniae), giả sương mai (Pseudoperonospora cubensis), phấn trắng (Erysiphe cichoracearum), héo xanh (Pseudomonas solanacearum), xì mủ
(Mycosphaerella melonis). Mức độ nhiễm bệnh của các THL tương đối thấp ở mức 5 đến $10 \%$. Trong số 21 THL và 1 giống đối chứng (TL3) thì có 12 THL (H5.6 x H34.6; H5.6 x H58.6; H5.6 x H77.6; H32.6 x H41.6; H32.6 x H53.6; H34.6 x H41.6; H34.6 x H58.6; H41.6 x H58.6; H41.6 x H77.6; H53.6 x H77.6 và H58.6 x H77.6) không bị nhiễm bệnh, 10 THL còn lại có tỷ lệ nhiễm bệnh từ 5 - 10\%, trong đó 2 THL H5.6 x H32.6 và H34.6 x H77.6 có tỷ lệ nhiễm bệnh thối thân; 3 THL H32.6 x H34.6; H32.6 x H58.6 và H41.6 x H53.6 có tỷ lệ nhiễm bệnh xì mủ và H5.6 x H41.6 có tỷ lệ 
nhiễm bệnh sương mai tương đối cao (10\%), các THL còn lại bị nhiễm bệnh thối thân (THL H5.6 x H53.6; H32.6 x H77.6 và H53.6 x H58.6), sương mai (THL H34.6 x H53.6), bệnh phấn trắng (THL $\mathrm{H} 5.6$ x H53.6 và H32.6 x H58.6) và héo xanh (THL H5.6 x H32.6 và H53.6 x H58.6) ở mức nhiễm bệnh $5 \%$.

\section{2. Đánh giá khả năng kết hợp riêng của 21 THL từ bảy dòng dưa lưới thế hệ $\mathrm{I}_{6}$}

Khả năng phối hợp chung của dòng (hoặc giống) (GCA) thể hiện hiệu ứng cộng tính, khả năng phối hợp riêng (SCA) thể hiện hiệu ứng phi cộng tính của các gen trong việc kiểm soát di truyền của một tính trạng (Ferreira \& ctv., 2004). Năng suất là một trong những yếu tố quyết định trong việc chọn tạo giống nói chung trong đó có dưa lưới. Đối với tính trạng năng suất, Abadia \& ctv. (1985) chỉ ra rằng, năng suất kiểm soát bởi hiệu ứng cộng tính và phi cộng tính, nhưng chủ yếu hiệu ứng phi cộng tính.

Về năng suất thực thu, kết quả Bảng 3 cho thấy, có 10/21 THL có giá trị khả năng phối hợp riêng theo hướng dương (SCA > 0), trong đó có 5/10 tổ hợp có giá trị SCA cao, đó là H5.6 x H32.6; H5.6 x H34.6.; H41.6 x H58.6; H53.6 x H77.6; H34.6 x H77.6. Trong đó, dòng sử dụng mẹ là dòng H5.6 có khả năng kết hợp riêng cao với dòng $\mathrm{H} 32.6$ và 34.6; dòng H77.6 sử dụng làm dòng bố có khả năng kết hợp riêng cao với dòng H53.6 và H34.6. Các dòng có khả năng kết hợp riêng cao cho lợi thế về năng suất đối với THL được tạo thành. Kết quả này cũng tương đồng với nghiên cứu của Anne và ctv. (2011), dòng có khả năng kết hợp riêng cao có ý nghĩa lớn đối với chọn giống dưa lưới.

Đối với hàm lượng chất rắn hòa tan (độ brix) (Bảng 4), có một số tranh cãi về việc kiểm soát di truyền. Một số tác giả nhận thấy rằng, có sự kiểm soát của hiệu ứng cộng tính và phi cộng tính (Cuarteiro \& ctv., 1985; Singh \& Randhawa, 1990); những người khác thì cho rằng, phi cộng tính không ảnh hưởng đáng kể (Zalapa \& ctv., 2006; Paris \& ctv., 2008). Monforte \& ctv. (2004) đã mô tả chi tiết các kết quả trái ngược nhau ở dưa lưới đối với chỉ tiêu này, tác giả khẳng định, hiệu ứng phi cộng tính có ảnh hưởng trong phép lai.

Kết quả đánh giá khả năng phối hợp riêng về độ brix của 7 dòng dưa lưới I6 cho thấy, có 11/21 THL có giá trị khả năng phối hợp riêng theo hướng dương (SCA > 0), trong đó có 5/11 tổ hợp có giá trị SCA cao, đó là H5.6 x H34.6; H32.6 x H41.6; H41.6 x H58.6; H41.6 x H77.6 và H53.6 x H58.6. Trong đó, dòng sử dụng mẹ là dòng H41.6 có khả năng kết hợp riêng cao về độ brix với dòng H58.6 và H77.6; dòng H58.6 sử dụng làm dòng bố có khả năng kết hợp riêng cao với dòng H53.6 và H41.6. Các dòng có khả năng kết hợp riêng cao cho lợi thế về độ brix đối với THL được tạo thành. Kết quả nghiên cứu cho thấy tính trạng năng suất và độ brix chịu ảnh hưởng của hiệu ứng phi cộng tính, điều này phù hợp với kết quả nghiên cứu của Monforte \& ctv. (2004) và Doan \& ctv. (2016).

\section{3. Đánh giá ưu thế lai của 21 THL dưa lưới}

Kết quả ở Bảng 5 đánh giá ưu thế lai trung bình (HM\%) được so sánh với giá trị trung bình năng suất thực thu và độ brix của bố và mẹ, các THL có ưu thế lai về năng suất thực thu dao động trong khoảng từ $24,7 \%$ đến $144,0 \%$ và độ brix từ (-) 19,3\% đến $(+) 34,7 \%$.

Ưu thế lai thực (HB\%) được so sánh với giá trị năng suất thực thu và độ brix của bố và mẹ tốt nhất, các THL có ưu thế lai thực về năng suất thực thu dao động trong khoảng từ $1,3 \%$ đến $6,1 \%$ và độ brix từ (-) $6,2 \%$ đến $(+) 10,1 \%$.

Ưu thế lai chuẩn (HS\%) được đánh giá dựa trên việc so sánh năng suất và độ brix của các THL với năng suất và độ brix của giống đối chứng. Các THL có ưu thế lai chuẩn về năng suất dao động trong khoảng từ (-) 21,9\% đến $(+) 24,9 \%$ và độ brix từ (-) 26,9 đến $(+)$ 14,4\%.

Như vậy, qua đánh giá ưu thế lai về năng suất cho thấy, có 2 THL thể hiện ưu thế lai về năng suất cao (cao hơn $10 \%$ so với đối chứng): H41.6 x H58.6 (24,9\%) và H53.6 x H77.6 (17,1\%); Có 3 THL thể hiện ưu thế lai về độ brix cao (cao hơn $10 \%$ so với đối chứng): H5.6 x H34.6 (11,5\%), H32.6 x H41.6 (14,4\%), H53.6 x H58.6 (11,5\%).

Tổ hợp lai H32.6 x H41.6 tuy có ưu thế lai về năng suất thấp (1,0\%), năng suất thực thu $(3,09$ tấn $/ 1000 \mathrm{~m}^{2}$ ) khác biệt không có ý nghĩa thống kê so với đối chứng (3,06 tấn/1000 m²), nhưng có ưu thế lại cao về độ brix cao hơn đối chứng $(14,4 \%)$. THL H53.6 x H58.6 cũng có kết quả tương tự.

Tổ hợp lai H41.6 x H58.6 tuy có ưu thế lai về độ brix thấp $(3,8 \%)$, độ brix $(13,50 \%)$ khác biệt không có ý nghĩa thống kê so với đối chứng $(13,00 \%)$, nhưng có ưu thế lại cao về năng suất 
Bảng 3. Đánh khả năng phối hợp riêng (SCA) về năng suất thực thu (tấn/1000 $\mathrm{m}^{2}$ ) của 7 dòng dưa lưới $\mathrm{I}_{6}$

\begin{tabular}{ccccccccc}
\hline$\sigma^{7}$ & H5.6 & H32.6 & H34.6 & H41.6 & H53.6 & H58.6 & H77.6 & \multirow{2}{*}{ GCA $^{1}$} \\
\cline { 2 - 6 } & & & & SCA & & & & \\
\hline H5.6 & 0,41 & 0,35 & $-0,14$ & $-0,21$ & $-0,24$ & $-0,17$ & $-0,12$ \\
H32.6 & & 0,04 & 0,05 & $-0,03$ & 0,02 & $-0,48$ & $-0,09$ \\
H34.6 & & & $-0,41$ & $-0,14$ & $-0,05$ & 0,22 & $-0,19$ \\
H41.6 & & & & $-0,17$ & 0,51 & 0,15 & 0,15 \\
H53.6 & & & & & 0,01 & 0,54 & 0,09 \\
H58.6 & & & & & & $-0,25$ & 0,18 \\
H77.6 & & & & & & & $-0,02$ \\
\hline
\end{tabular}

${ }^{1}$ GCA: năng phối hợp chung.

Bảng 4. Đánh khả năng phối hợp riêng (SCA) về độ brix của 7 dòng dưa lưới $\mathrm{I}_{6}$

\begin{tabular}{|c|c|c|c|c|c|c|c|c|}
\hline $0^{x}$ & H5.6 & H32.6 & H34.6 & H41.6 & H53.6 & H58.6 & H77.6 & \multirow{2}{*}{$\mathrm{GCA}^{1}$} \\
\hline 우 & \multicolumn{7}{|c|}{ SCA } & \\
\hline $\begin{array}{l}\mathrm{H} 5.6 \\
\end{array}$ & & $-0,06$ & $\overline{2,71}$ & $\overline{0,34}$ & $\begin{array}{c}-0,24 \\
\end{array}$ & $\begin{array}{l}-1,79 \\
\end{array}$ & $\begin{array}{c}-0,96 \\
\end{array}$ & $\overline{0,90}$ \\
\hline H32.6 & & & $-1,15$ & 3,01 & 0,50 & $-0,65$ & $-1,65$ & $-0,54$ \\
\hline H34.6 & & & & $-3,35$ & 0,40 & 0,52 & 0,86 & 0,46 \\
\hline H41.6 & & & & & $-2,80$ & 1,15 & 1,65 & 0,16 \\
\hline H53.6 & & & & & & 1,40 & 0,74 & 0,91 \\
\hline H58.6 & & & & & & & $-0,64$ & $-0,04$ \\
\hline H77.6 & & & & & & & & $-0,04$ \\
\hline
\end{tabular}

${ }^{1}$ GCA: năng phối hợp chung.

Bảng 5. Đánh giá ưu thế lai về năng suất và độ brix của 21 tổ hợp lai từ 7 dòng dưa lưới thế hệ I6

\begin{tabular}{ccccccc}
\hline Cặp lai & \multicolumn{3}{c}{ Năng suất } & \multicolumn{3}{c}{ Độ brix } \\
\cline { 2 - 7 }$\left(\sigma^{\pi}\right.$ × o)/dòng & HM (\%) & HB (\%) & HS (\%) & HM (\%) & HB (\%) & HS (\%) \\
\hline H5.6 x H32.6 & 75,8 & 3,7 & 4,4 & $-12,5$ & $-4,2$ & $-17,4$ \\
H5.6 x H34.6 & 70,9 & 3,4 & $-1,3$ & 34,7 & 10,1 & 11,5 \\
H5.6 x H41.6 & 87,8 & 3,7 & $-5,9$ & $-0,8$ & $-0,3$ & $-9,0$ \\
H5.6 x H53.6 & 48,1 & 2,4 & $-10,5$ & 2,0 & 0,6 & $-7,7$ \\
H5.6 x H58.6 & 62,1 & 2,9 & $-8,2$ & $-19,3$ & $-6,2$ & $-26,9$ \\
H5.6 x H77.6 & 48,3 & 2,4 & $-12,7$ & $-4,0$ & $-1,2$ & $-20,5$ \\
H32.6 x H34.6 & 45,5 & 2,3 & $-10,5$ & $-5,7$ & $-1,8$ & $-15,4$ \\
H32.6 x H41.6 & 87,3 & 3,9 & 1,0 & 15,8 & 5,5 & 14,4 \\
H32.6 x H53.6 & 50,0 & 2,7 & $-3,6$ & 3,4 & 1,2 & 0,8 \\
H32.6 x H58.6 & 67,0 & 3,4 & 1,0 & $-13,2$ & $-4,5$ & $-15,4$ \\
H32.6 x H77.6 & 24,7 & 1,3 & $-21,9$ & $-14,3$ & $-4,5$ & $-23,1$ \\
H34.6 x H41.6 & 58,1 & 2,5 & $-17,3$ & $-18,3$ & $-5,8$ & $-26,9$ \\
H34.6 x H53.6 & 43,0 & 2,2 & $-10,5$ & 22,1 & 6,9 & 7,7 \\
H34.6 x H58.6 & 61,9 & 3,0 & $-4,8$ & 14,8 & 4,6 & 1,3 \\
H34.6 x H77.6 & 59,8 & 3,0 & $-2,5$ & 29,0 & 8,2 & 3,8 \\
H41.6 x H53.6 & 81,4 & 3,7 & $-0,2$ & $-14,2$ & $-4,7$ & $-19,2$ \\
H41.6 x H58.6 & 144,0 & 6,1 & 24,9 & 10,4 & 3,4 & 3,8 \\
H41.6 x H77.6 & 99,8 & 4,4 & 6,6 & 24,6 & 7,5 & 7,7 \\
H53.6 x H58.6 & 73,3 & 3,8 & 6,6 & 17,3 & 5,8 & 11,5 \\
H53.6 x H77.6 & 83,8 & 4,4 & 17,1 & 21,7 & 6,7 & 6,4 \\
H58.6 x H77.6 & 57,3 & 2,9 & $-5,8$ & 1,8 & 0,5 & $-11,5$ \\
\hline
\end{tabular}

Ưu thế lai trung bình (HM\%), Ưu thế lai thực (HB\%), Ưu thế lai chuẩn (HS\%). 
cao hơn đối chứng (14,4\%). THL H53.6 x H77.6 cũng có kết quả tương tự.

\section{Kết Luận}

Đánh giá khả năng phối hợp riêng dựa trên tính trạng năng suất và độ brix của 7 dòng dưa lưới tự phối I6 (H5.6, H32.6, H34.6, H41.6, H53.6, H58.6, H77.6) bằng phương pháp lai luân giao (mô hình Griffing 4) cho thấy rằng, các dòng H41.6 và H58.6, H53.6 và H77.6 có khả năng phối hợp riêng cao về tính trạng năng suất; các dòng H5.6 và H34.6, H32.6 và H41.6, H53.6 và $\mathrm{H} 58.6$ có khả năng phối hợp riêng cao về tính trạng brix.

Chọn được 04 THL triển vọng có khả năng phối hợp riêng cao và có ưu thế lai cao vượt giống đối chứng từ $10 \%$ trở lên phục vụ công tác sản suất gồm tổ hợp lai H41.6 x H58.6, H53.6 x H77.6, H32.6 x H41.6 và H53.6 x H58.6.

\section{Tài Liệu Tham Khảo (References)}

Abadia, J., Cuarteiro, M. L. G. J., \& Nuez, F. (1985). Herencia de caracteres cuantitativos en melón. Instituto Nacional de Investigación y Tecnología Agraria y Alimentaria 28, 83-91.

AHRD (High-tech Agricultural Reasearch and Development Center). (2015). Growing plants in substratesin a net house. Ho Chi Minh City, Vietnam: AHRD.

Anne, K., Glauber, H., Manoel, A., Elaíne, W., \& José, H. (2011). Diallel analysis of yield and quality traits of melon fruits. Crop Breeding and Applied Biotechnology 11(4), 313-319.

Cuarteiro, M. L. G. J., Abadia, J., \& Nuez, F. (1985). Herencia de caracteres cualitativos en melón. Instituto Nacional de Investigación y Tecnología Agraria y Alimentaria 28, 72-82.

Doan, C. H., Nguyen, H. P., Ha, L. T., Duong, X. H., \& Phan, Q. D. (2016). Combining ability of six S5 inbred lines of melon (Cucumis melon L.). Vietnam Journal of Agricultural Sciences 11(72), 50-54.

Ferreira, F. M., Ribeiro Júnior, J. I., Pacheco, C. A. P., Silva, C. H. O., \& Martins Filho, S. (2004). Genetic components of combining ability in a complete diallel. Crop Breeding and Applied Biotechnology 4(3), 338-343.
Griffing, B. (1956). Concept of general and specific combining ability in relation to diallen crossing systems. Australian Journal of Biological Sciences 9(4), 463493.

Huynh, T. Q., Hoang, H. D., \& To, T. T. T. (2019). A study of selecting materials and cultivars of Muskmelon (Cucumis melo L.) suitable for the southern region of Vietnam ( $4^{\text {th }}$ year). Research and Development Center for Hi-tech Agriculture, Ho Chi Minh City, Vietnam.

MARD (Ministry of Agriculture and Rural Development). (2014). Decision No. 512 /QD-TTCLT dated on November 19, 2014. The Process of Muskmelon (Cucumis melo L.) cultivation. Ha Noi, Vietnam: MARD Office.

MARD (Ministry of Agriculture and Rural Development). (2003). Decision No. 10TCN 224:2003 dated on 2003. Surveillance method of plant pests. Ha Noi, Vietnam: MARD Office.

Monforte, A. J., Oliver, M., Gonzalo, M. J., Alvarez, J. M., DolcetSanjuan, R., \& Arús, P. (2004). Identification of quantitative trait lociinvolved in fruit quality traits in melon (Cucumis melo L.). Theoretical and Applied Genetics 108(4), 750-758.

Ngoc, Q. (2020). Replicating the model of planting the Muskmelon. Retrieved August 10, 2020, from https://congthuong.vn/dua-luoi-cong-nghe-caomo-hinh-can-nhan-rong-140939.html.

Paris, M. K., Zalapa, J. E., McCreight, J. D., \& Staub, J. E. (2008). Genetic dissection of fruit quality components in melon (Cucumis melo L.) using a RIL population derived from exotic x elite US Western Shipping germplasm. Molecular Breeding 22(3), 405-419.

Phan, K. T. (2006). Plant breeding curriculum. Ho Chi Minh City, Vietnam: Agricultural Publishing House.

Singh, M. J., \& Randhawa, K. S. (1990). Evaluation of heterosis and ability for traits in muskmelon. Indian Journal Horticulture 47(2), 228-232.

Zalapa, J. E., Staub, J. E., \& McCreight, J. D. (2006). Generation means analysis of plant architectural traits and fruit yield in melon. Plant Breeding 125(5), 482487. 